SUPPORTING INFORMATION

\title{
Roles of Sterol Derivatives in Regulating the Properties of Phospholipid Bilayer Systems
}

\author{
Tham Thi Bui, Keishi Suga, Hiroshi Umakoshi*
}

Division of Chemical Engineering, Graduate School of Engineering Science, Osaka University

1-3 Machikaneyama, Toyonaka, Osaka, 560-8531, Japan.

* Corresponding author:

Prof. Dr. Hiroshi Umakoshi

Phone: +81-6-6850-6287

E-mail: b-ice@cheng.es.osaka-u.ac.jp 

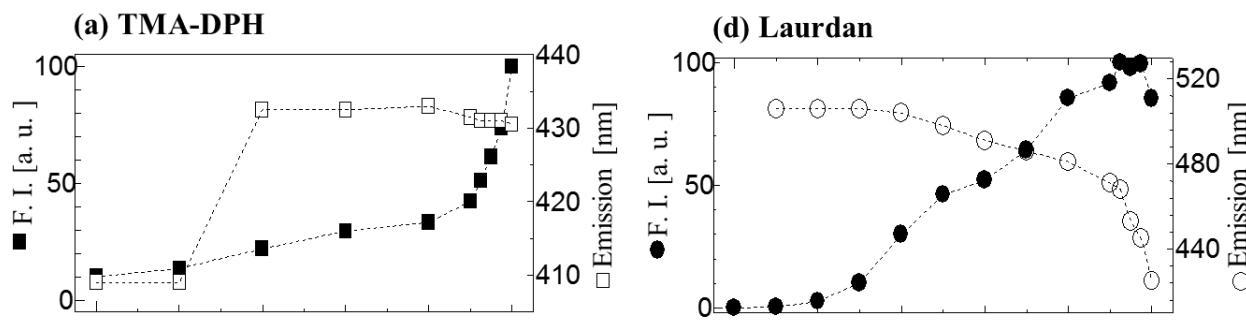

(g) Dielectric constant
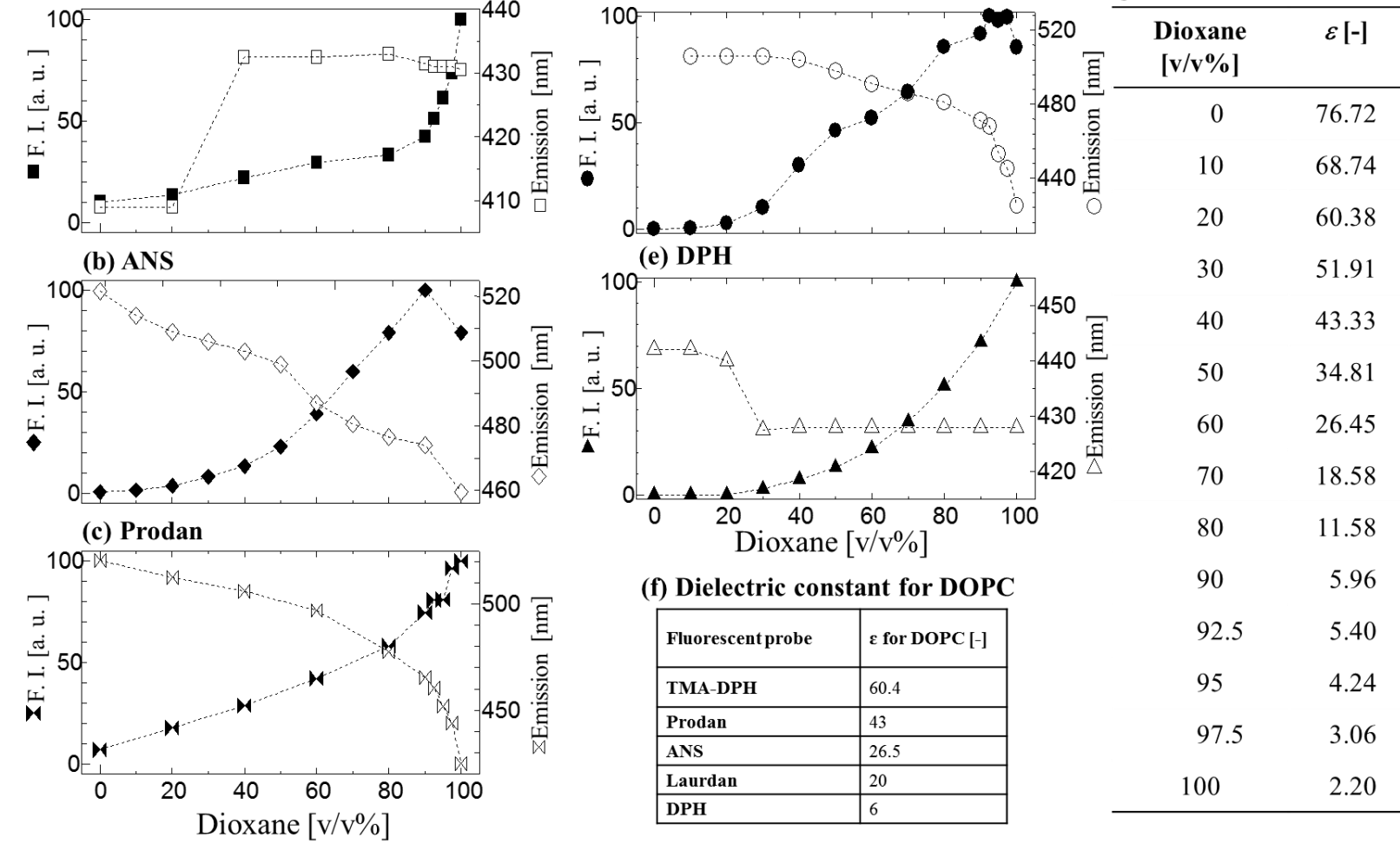

60.38

$30 \quad 51.91$

$40 \quad 43.33$

$50 \quad 34.81$

$60 \quad 26.45$

$\begin{array}{ll}70 & 18.58\end{array}$

$80 \quad 11.58$

$90 \quad 5.96$

$92.5 \quad 5.40$

$95 \quad 4.24$

$97.5 \quad 3.06$

$100 \quad 2.20$

Figure S1. Characterization of fluorescent probes in 1,4-dioxane/water systems: (a) TMA-DPH, (b) ANS, (c) Prodan, (d) Laurdan, and (e) DPH. The estimated dielectric environment around each probe in DOPC liposome is summarized in Table (f). The dielectric constants of solvents were controlled by mixing 1,4-dioxane and water with different volume ratio $(\mathrm{g}) .{ }^{1}$ Fluorescent probes such as TMA-DPH, ANS, Prodan, Laurdan, and DPH are sensitive to their surrounding environments, and are used as the probes for liposome membranes. Liposome membranes have a hydrophobicity gradient in the vertical direction of membrane,${ }^{2}$ which is reflected by binding depths of fluorescent probes. In 1,4-dioxane/water systems, each probe showed different emission wavelength and fluorescent intensity. ${ }^{3-6}$ Thus, the emission wavelength and intensity in liposome membranes were also depended on the surrounding environment. By analyzing the emission property of each probe in 1,4-dioxane/water systems, the location of probe in the liposome was estimated on the basis of (i) emission wavelength and (ii) intensity.

(i) Laurdan, ANS, Prodan: These probes showed the emission peak shifts depending on the solvent hydrophobicity. Thus, their locations can be estimated by analyzing the emission peak wavelength in liposomes. For ANS, only one emission peak could be seen at $484 \mathrm{~nm}$, which means that ANS bound to the similar location in membrane with different amounts, 
thus the surface hydrophobicity (i.e. the exposure of hydrophobic core to water) can be investigated by $I_{484}$. For Laurdan and Prodan, the appearance of two peaks (also see Figures S3 and S6) indicate that the location of probe altered up on the membrane properties. The $G P_{340}$ values can be used to understand the polarity of the membrane (see supporting information Figure S2).

(ii) DPH, TMA-DPH: These probes have the emission peaks at $430 \mathrm{~nm}$, independent to the surrounding hydrophobicity. In the liposome systems, both DPH and TMA-DPH showed similar emission peak wavelength a $430 \mathrm{~nm}$, indicating that DPH and TMA-DPH located at $\varepsilon<43$ and $\varepsilon<60$, respectively. The fluorescence intensity increased in proportion to the hydrophobicity of solvent. In the case of DPH inserted into liposome membranes, the emission peak at $428 \mathrm{~nm}$ and its fluorescence intensity is ca. 70\% as compared to 1,4dioxane $100 \%$ as solvent, indicating that DPH is inserted into hydrophobic regions $(\varepsilon \sim 6)$. In the same manner, TMA-DPH can be located at $\varepsilon \sim 60$.

Considering our experimental results ${ }^{3-6}$ and literatures, ${ }^{7-10}$ the location of each probe in DOPC liposome can be estimated (f). No peak shifts of ANS or TMA-DPH were observed in DOPC/sterol liposomes, it was assumed that the binding location of probes did not change, and that the in situ environment around probes could be altered depending on the type and amount of sterols. Hence, the variation in the membrane properties could be provided by the different interaction between DOPC and sterol. The location of Chl, Lan, Erg might be similar. The hydration in the membrane surface would be sensitive to the chemical structure of sterol and its interaction with DOPC. 

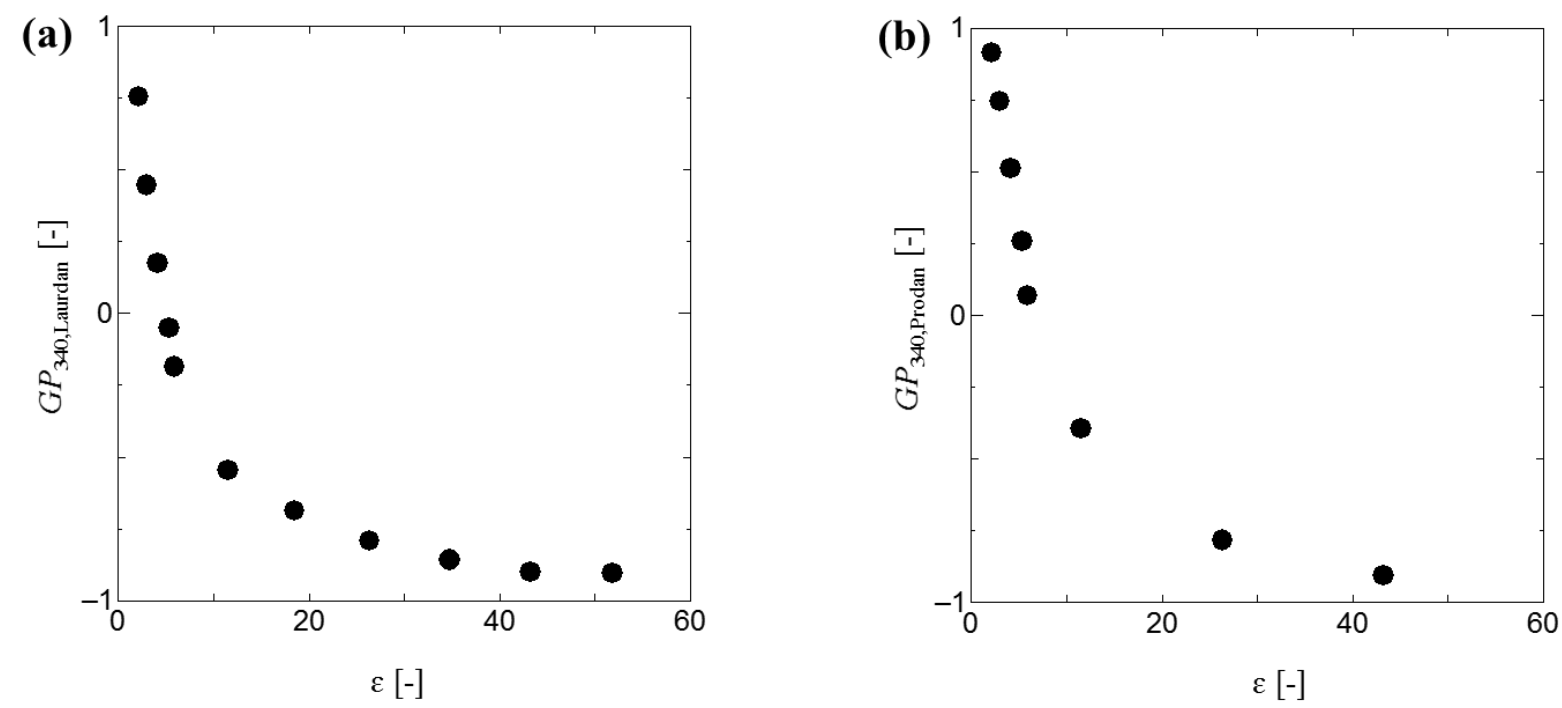

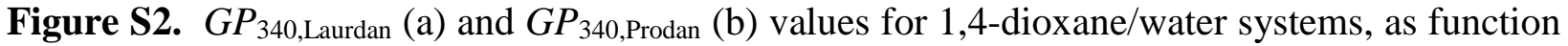
of $\varepsilon$ values. In 1,4-dioxane/water systems, the $G P_{340, \text { Laurdan }}$ and $G P_{340 \text {,Prodan }}$ values were monotonically decreased by increasing $\varepsilon$ values. Thus, the $G P_{340}$ value analysis can be applied to monitor the surrounding of Laurdan or Prodan depending on the polar environments in the membrane. Considering the peak shift of Laurdan or Prodan, its actual location is flexible and depends on the membrane phase state, while it surely settles down in the inner membrane because it showed much higher intensity as compared to water system. 


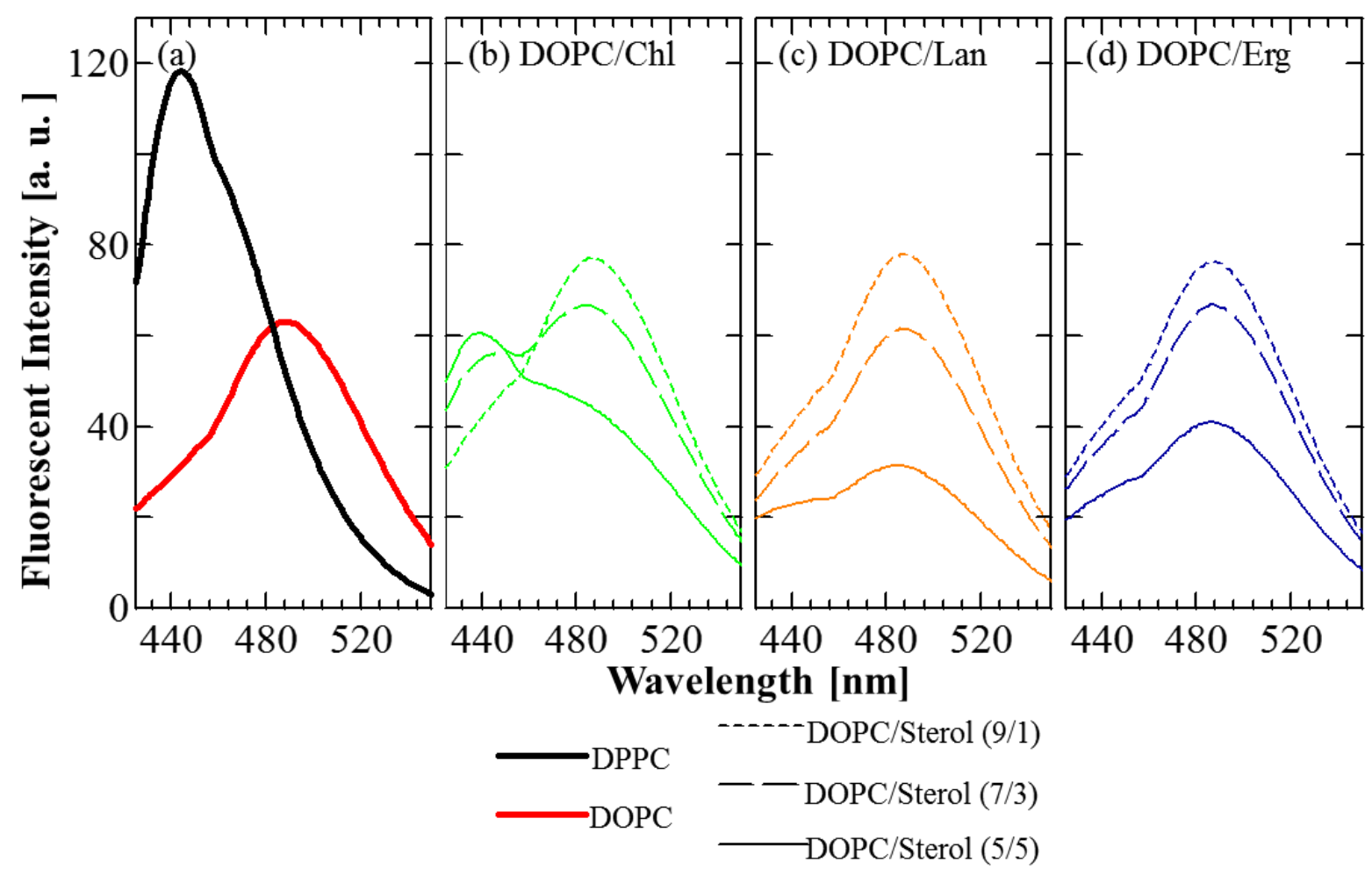

Figure S3. Fluorescent spectra of Laurdan for DOPC and DPPC liposomes (a), for DOPC/Chl liposomes (b), DOPC/Lan liposomes (c), and DOPC/Erg liposomes (d). The results indicated that fluorescent intensity of DOPC/Chl from 0 to $50 \mathrm{~mol} \%$ changed with peak shifts from 490 into 440 $\mathrm{nm}$. It means that $\mathrm{Chl}$ at $10 \mathrm{~mol} \%$ remained membrane in liquid disordered phase, while $30 \mathrm{~mol} \%$ of Chl expressing both peaks at these wavelengths fixed membrane in heterogeneous phase, and $50 \mathrm{~mol} \%$ of Chl changed membrane into ordered phase. In the cases of Lan and Erg, all of them showed the peaks at $490 \mathrm{~nm}$, suggesting that they were in homogeneous disordered phases similar to DOPC. 

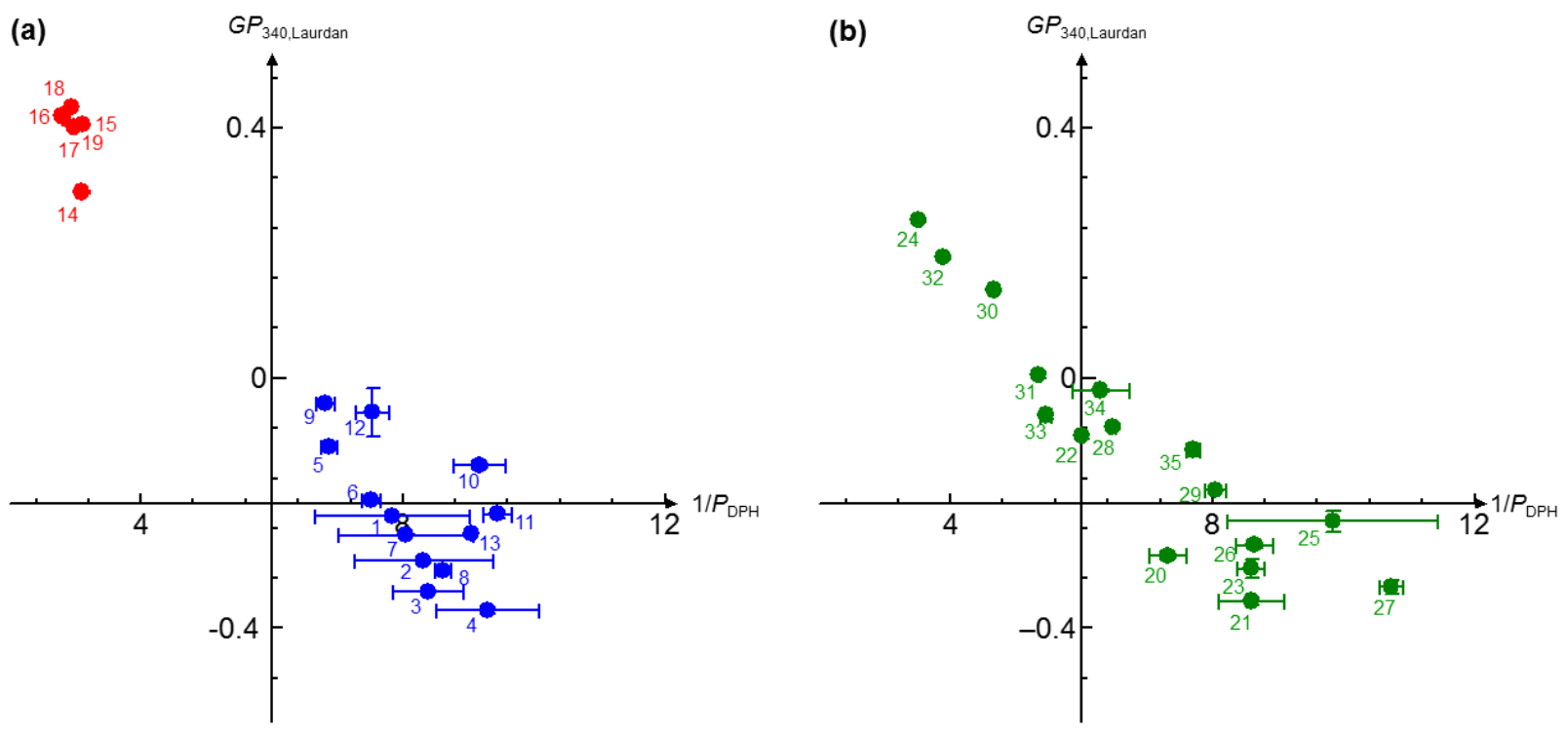

Figure S4. Cartesian diagram for homogeneous liposome systems (a) and for heterogeneous liposome systems (b). Membrane fluidities (1/P $P_{\mathrm{DPH}}, x$ axis) and membrane polarities ( $G P_{340, \mathrm{Laurdan}}$, $y$ axis) were plotted for various liposomes as shown in Table S1. Based on our previous reports, ${ }^{11,12}$ it was found that by using DPH fluorescent probe, the $1 / P$ value for DPPC liposome between $l_{\mathrm{d}}$ and $s_{\mathrm{o}}$ phases is estimated to be $1 / P_{\mathrm{DPH}}=6$, while its membrane polarity determined by Laurdan shown that the threshold between disorder and order phases is $G P_{340, \text { Laurdan }}=-0.2$. Therefore, the threshold point in the Cartesian diagram is considered with $1 / P_{\mathrm{DPH}}=6.0$ and $G P_{340, \text { Laurdan }}=-0.2 .{ }^{10}$ Obviously, the $l_{\mathrm{d}}$-phase liposomes appeared in the $1^{\text {st }}$ and $4^{\text {th }}$ quadrant $\left(1 / P_{\mathrm{DPH}}>6\right)$, while the $s_{0^{-}}$ phase liposomes appeared in the $2^{\text {nd }}$ quadrant $\left(1 / P_{\mathrm{DPH}}<6\right)$. The $G P_{340, \text { Laurdan }}$ values were $>0$ under $T_{\mathrm{m}}$, and $<0$ above $T_{\mathrm{m}}$. When a liposome shows similar properties to that in $l_{\mathrm{d}}$ phase, it could be in $l_{\mathrm{d}}$ phase (disordered). In similar manner, a liposome possessing similar properties to that in $s_{\mathrm{o}}$ phase, it could be in $s_{\mathrm{o}}$ phase (ordered). For heterogeneous liposome systems (green symbols plotted in (b)), some liposomes appeared between $l_{\mathrm{d}}$-phase and $s_{\mathrm{o}}$-phase clusters, indicating that they were in heterogeneous phases $\left(l_{\mathrm{d}}+l_{\mathrm{o}}, l_{\mathrm{d}}+s_{0}\right.$, etc. $)$. It is notable that $1 / P_{\mathrm{DPH}}$ and $G P_{340, \text { Laurdan }}$ values could be insensitive to lipid/probe molar ratio (at least the range between 20/1 to 1000/1), indicating that the membrane properties evaluated by DPH and Laurdan can reflect each phase behavior. 
Table S1. Summary of the phase state and membrane properties for liposomes.

\begin{tabular}{|c|c|c|c|c|c|c|}
\hline \multirow{2}{*}{\multicolumn{2}{|c|}{$\begin{array}{l}\text { composition } \\
\text { (homogeneous system, } l_{\mathrm{d}} \text { phase) }\end{array}$}} & \multicolumn{2}{|c|}{$T\left[{ }^{\circ} \mathrm{C}\right]$} & \multirow[t]{2}{*}{ phase state ${ }^{* a}$} & \multirow[t]{2}{*}{$1 / P_{\mathrm{DPH}}$} & \multirow{2}{*}{$G P_{340, \text { Laurdar }}$} \\
\hline & & & & & & \\
\hline 1 & DOPC & 22 & $\left(>T_{\mathrm{m}}\right)$ & $l_{\mathrm{d}}$ & 7.84 & -0.22 \\
\hline 2 & DOPC & 30 & $\left(>T_{\mathrm{m}}\right)$ & $l_{\mathrm{d}}$ & 8.30 & -0.29 \\
\hline 3 & DOPC & 40 & $\left(>T_{\mathrm{m}}\right)$ & $l_{\mathrm{d}}$ & 8.39 & -0.34 \\
\hline 4 & DOPC & 50 & $\left(>T_{\mathrm{m}}\right)$ & $l_{\mathrm{d}}$ & 9.29 & -0.37 \\
\hline 5 & POPC & 19 & $\left(>T_{\mathrm{m}}\right)$ & $l_{\mathrm{d}}$ & 6.88 & -0.11 \\
\hline 6 & POPC & 30 & $\left(>T_{\mathrm{m}}\right)$ & $l_{\mathrm{d}}$ & 7.52 & -0.19 \\
\hline 7 & POPC & 40 & $\left(>T_{\mathrm{m}}\right)$ & $l_{\mathrm{d}}$ & 8.05 & -0.25 \\
\hline 8 & POPC & 50 & $\left(>T_{\mathrm{m}}\right)$ & $l_{\mathrm{d}}$ & 8.62 & -0.30 \\
\hline 9 & DMPC & 30 & $\left(>T_{\mathrm{m}}\right)$ & $l_{\mathrm{d}}$ & 6.82 & -0.04 \\
\hline 10 & DMPC & 40 & $\left(>T_{\mathrm{m}}\right)$ & $l_{\mathrm{d}}$ & 9.17 & -0.13 \\
\hline 11 & DMPC & 50 & $\left(>T_{\mathrm{m}}\right)$ & $l_{\mathrm{d}}$ & 9.44 & -0.21 \\
\hline 12 & DPPC & 50 & $\left(>T_{\mathrm{m}}\right)$ & $l_{\mathrm{d}}$ & 7.53 & -0.05 \\
\hline 13 & bSM & 50 & $\left(>T_{\mathrm{m}}\right)$ & $l_{\mathrm{d}}$ & 9.04 & -0.24 \\
\hline \multicolumn{7}{|c|}{ (homogeneous system. $s_{\mathrm{o}}$ phase) } \\
\hline 14 & DMPC & 19 & $\left(<T_{\mathrm{m}}\right)$ & $s_{\mathrm{o}}$ & 3.10 & 0.29 \\
\hline 15 & DPPC & 22 & $\left(<T_{\mathrm{m}}\right)$ & $s_{\mathrm{o}}$ & 2.82 & 0.42 \\
\hline 16 & DPPC & 30 & $\left(<T_{\mathrm{m}}\right)$ & $s_{\mathrm{o}}$ & 2.79 & 0.41 \\
\hline 17 & DPPC & 40 & $\left(<T_{\mathrm{m}}\right)$ & $s_{\mathrm{o}}$ & 2.97 & 0.40 \\
\hline 18 & DSPC & 25 & $\left(<T_{\mathrm{m}}\right)$ & $s_{\mathrm{o}}$ & 2.95 & 0.43 \\
\hline 19 & bSM & 25 & $\left(<T_{\mathrm{m}}\right)$ & $s_{\mathrm{O}}$ & 3.11 & 0.40 \\
\hline \multicolumn{7}{|c|}{ (heterogeneous system) } \\
\hline 20 & $\mathrm{DOPC} / \mathrm{DPPC}=(75 / 25)$ & 30 & & & 7.32 & -0.28 \\
\hline 21 & DOPC/DPPC=(75/25) & 50 & & $\left(l_{\mathrm{d}}\right)$ & 8.59 & -0.35 \\
\hline 22 & $\mathrm{DOPC} / \mathrm{DPPC}=(50 / 50)$ & 30 & & & 6.01 & -0.09 \\
\hline 23 & $\mathrm{DOPC} / \mathrm{DPPC}=(50 / 50)$ & 50 & & $\left(l_{\mathrm{d}}\right)$ & 8.59 & -0.30 \\
\hline 24 & DOPC/DPPC= $=(25 / 75)$ & 30 & & & 3.51 & 0.25 \\
\hline 25 & DOPC/DPPC=(25/75) & 50 & & $\left(l_{\mathrm{d}}\right)$ & 9.83 & -0.22 \\
\hline 26 & $\mathrm{DOPC} / \mathrm{Ch}=(90 / 10)$ & 30 & & & 8.64 & -0.26 \\
\hline 27 & $\mathrm{DOPC} / \mathrm{Ch}=(90 / 10)$ & 50 & & & 10.72 & -0.33 \\
\hline 28 & $\mathrm{DOPC} / \mathrm{Ch}=(70 / 30)$ & 30 & & & 6.47 & -0.07 \\
\hline 29 & $\mathrm{DOPC} / \mathrm{Ch}=(70 / 30)$ & 50 & & & 8.04 & -0.17 \\
\hline 30 & $\mathrm{DOPC} / \mathrm{Ch}=(50 / 50)$ & 30 & & & 4.66 & 0.14 \\
\hline 31 & $\mathrm{DOPC} / \mathrm{Ch}=(50 / 50)$ & 50 & & & 5.34 & 0.004 \\
\hline 32 & $\mathrm{DOPC} / \mathrm{DPPC} / \mathrm{Ch}=(4 / 4 / 2)$ & 30 & & & 3.88 & 0.19 \\
\hline 33 & $\mathrm{DOPC} / \mathrm{DPPC} / \mathrm{Ch}=(4 / 4 / 2)$ & 50 & & & 5.45 & -0.06 \\
\hline 34 & $\mathrm{DOPC} / \mathrm{SM} / \mathrm{Ch}=(6 / 2 / 2)$ & 30 & & & 6.29 & -0.02 \\
\hline 35 & $\mathrm{DOPC} / \mathrm{SM} / \mathrm{Ch}=(6 / 2 / 2)$ & 50 & & & 7.70 & -0.11 \\
\hline
\end{tabular}

${ }^{*}$ Phase states are referred based on the literatures ${ }^{11,13-15}$ 


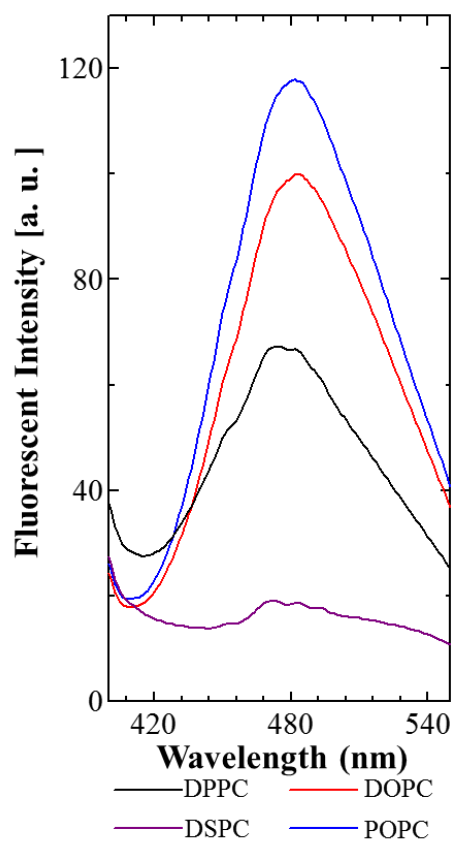

Figure S5. Relative fluorescence spectra of ANS for different liposomes at $37^{\circ} \mathrm{C}$. Because the fluorescence emission peak of ANS depends on the surrounding hydrophobicity, the ANS emission peak wavelength can be used as indicator to monitor the microscopic environment around ANS in the lipid membrane. Figure S5 shows the fluorescent spectra of ANS $37{ }^{\circ} \mathrm{C}$, for the liposomes in $l_{\mathrm{d}}$ phases (DOPC, POPC) and in $s_{\mathrm{o}}$ phases (DPPC, DSPC), where the emission intensity at $484 \mathrm{~nm}$ for DOPC liposome was defined as 100 [a.u.]. The results showed that all liposomes had the similar emission wavelengths (at $484 \mathrm{~nm}$ ) but differences in intensities. It means that the same location of ANS but the different amount of bound ANS in the membrane.

Actually, the ANS probes bound to liposomes in $l_{\mathrm{d}}$ phases showed higher intensities than those in $s_{\mathrm{o}}$ phases. The ANS intensity in water was negligible. The liposomes in $l_{\mathrm{d}}$ phases have lower lipid packing densities (mean headgroup area: $0.72 \mathrm{~nm}^{2} /$ molecule; more hydrophobic cavities), while the liposomes $s_{0}$ phases have higher lipid packing densities (mean headgroup area: 0.48 $\mathrm{nm}^{2} /$ molecule). ${ }^{11,13}$ Thus, the lipid packing is critical factor for the degree in the exposure of hydrophobic core to water, where ANS can be distributed to such hydrophobic sites. Consequently, the amount of bound ANS will be higher and leads to the higher fluorescence intensity.

It has been reported that the quantum yield $\phi$ of ANS in DPPC membrane ${ }^{16}$ and in water ${ }^{17}$ are $\phi=0.27$ and $\phi<0.01$, respectively. Considering the different binding amount of ANS in DOPC and 
DPPC liposomes, the quantum yield $\phi$ of ANS would be dependent on the membrane hydrophobicicty, I484. According to Uchiyama et al., the fluorescence quantum yields $\phi$ can be determined with the following equation: ${ }^{18}$

$$
\phi=\phi_{\mathrm{R}} \times(F / A) /\left(F_{\mathrm{R}} / A_{\mathrm{R}}\right) \times\left(n / n_{\mathrm{R}}\right)^{2},
$$

where $\phi_{\mathrm{R}}$ is the fluorescence quantum yield of a reference compound (anthracene, $\phi_{\mathrm{R}}=0.297$ in ethanol ${ }^{19}$ ). $F$ and $F_{\mathrm{R}}$ are the integrated values of fluorescence spectra for ANS and reference, $A$ and $A_{\mathrm{R}}$ are the absorbance at the excitation wavelength, $n$ and $n_{\mathrm{R}}$ are refractive indices of solvents. The estimated $\phi$ values of ANS in DOPC, in DPPC, and in PBS buffer were 0.285 \pm 0.008 , $0.260 \pm 0.005$, and $0.006 \pm 0.001$, respectively (at $37^{\circ} \mathrm{C}$ ). It is therefore investigated that the (apparent) quantum yield of ANS in DOPC was higher than that in DPPC liposome. 


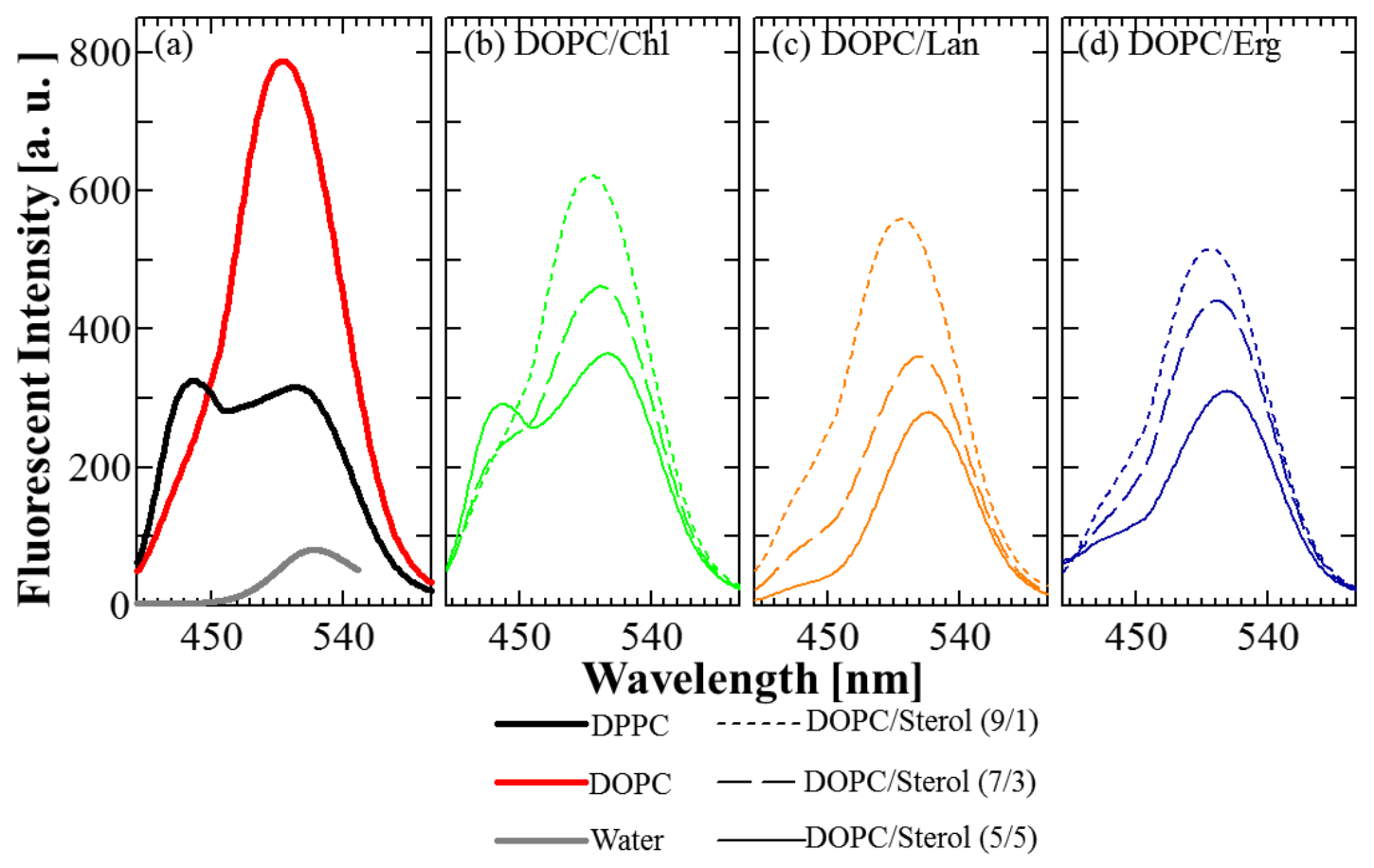

Figure S6. Fluorescent spectra of Prodan in DOPC, DPPC liposomes and water (a), in DOPC/Chl liposomes (b), DOPC/Lan liposomes (c), and DOPC/Erg liposomes (d). The fluorescent intensity of Prodan in water was lower enough, as compared to that in liposome systems. Thus, the partitioning of probe in water hardly influences on the Prodan spectra in the presence of liposomes. ${ }^{9}, 10$ 


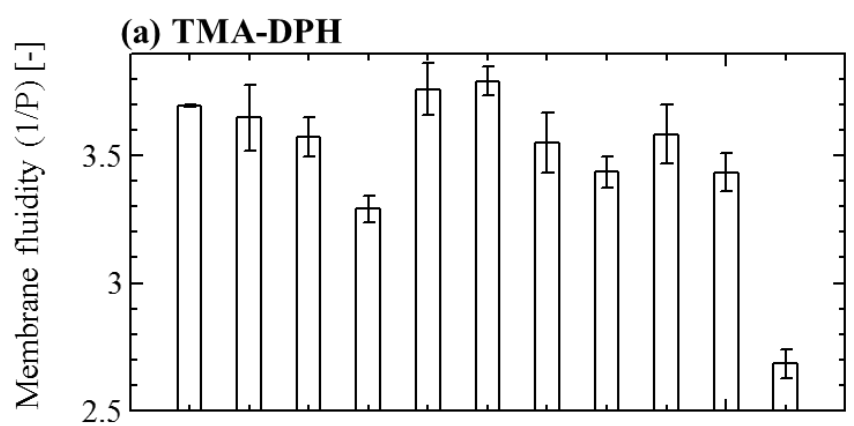

(b) ANS
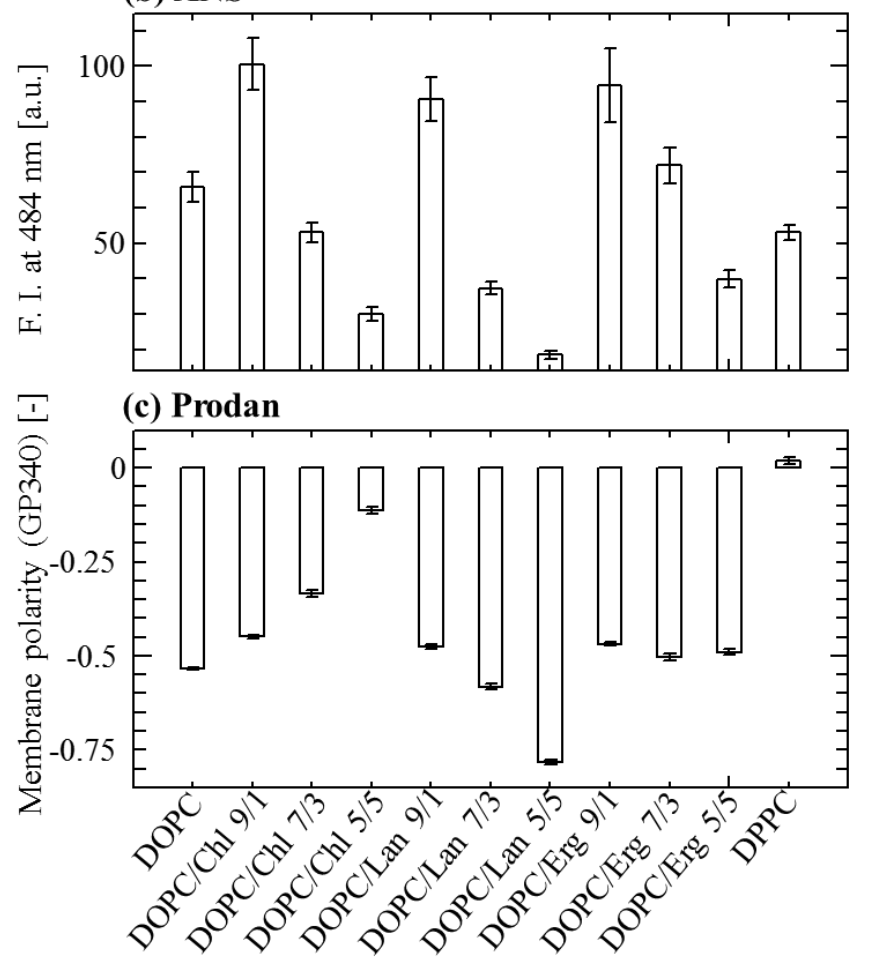

Figure S7. The raw data for membrane fluidity investigated by TMA-DPH (a), fluorescent intensity of ANS at $I_{484}$ (b) and membrane polarity analyzed by Prodan (d). 


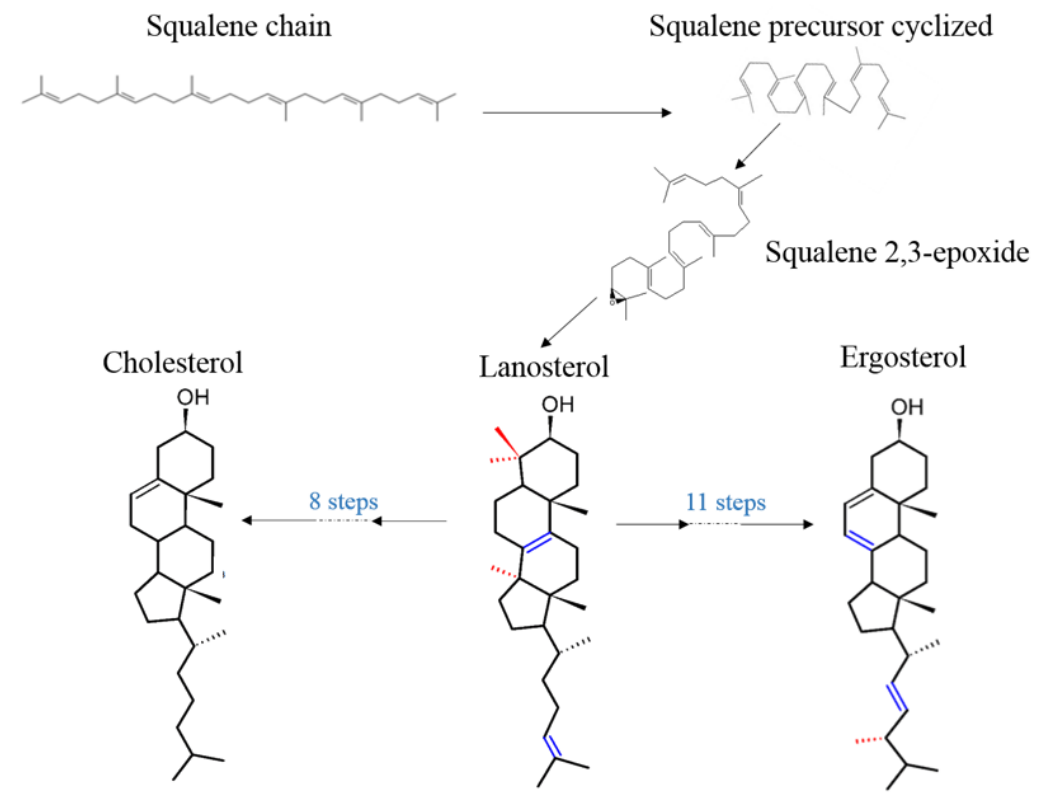

Figure S8. Modified biosynthesis pathway of sterols in membrane. ${ }^{20}$ 


\section{REFERENCES}

(1) Critchpield, F. E.; Gibson Jr., J. A.; Hall, J. L. Dielectric constant for the dioxane-water system from 20 to $35^{\circ}$. J. Am. Chem. Soc. 1953, 75, 1991-1992.

(2) Cevc, G. Membrane electrostatics. Biochim. Biophys. Acta. 1990, 1031, 311-382.

(3) Umakoshi, H.; Suga, K. Use Liposome as a Designable Platform for Molecular Recognition from "Statistical Separation" to "Recognitive Separation" . Solvent Extr. Res. Dev. Jpn. 2013, 20, 1-13.

(4) Ishigami, T.; Suga, K.; Umakoshi, H. Chiral Recognition of 1-Amino Acids on Liposomes Prepared with 1-Phospholipid, ACS Appl. Mater. Interf. 2015, 7 (38), 21065-21072.

(5) Suga, K.; Tanabe, T.; Umakoshi, H. Heterogeneous cationic liposomes modified with $3 \beta$-N$\left[\left(\mathrm{N}^{\prime}, \mathrm{N}^{\prime}\right.\right.$-dimethylamino)ethyl $]$ carbamoylcholesterol can induce partial conformational changes in messenger RNA and regulate translation in an escherichia coli cell-free translation system, Langmuir 2013, 29 (6), 1899-1907.

(6) Suga, K.; Tanabe, T.; Tomita, H.; Shimanouchi, T.; Umakoshi, H. Conformational change of single-stranded RNAs induced by liposome binding. Nucleic Acids Res. 2011, 39, 8891-8900.

(7) Parasassi, T.; Gratton, E. Membrane lipid domains and dynamics as detected by Laurdan fluorescence. J. Fluoresc. 1995, 5, 59-69.

(8) Dhanikula, A.B.; Panchagnula, R. Fluorescence anisotropy, FT-IR spectroscopy and 31-P NMR studies on the interaction of paclitaxel with lipid bilayers. Lipids 2008, 43 (6), 569-579.

(9) Krasnowska, E.K.; Gratton, E.; Parasassi, T. Prodan as a membrane surface fluorescence probe: Partitioning between water and phospholipid phases, Biophys. J. 1998, 74 (4), 19841993.

(10) Cwiklik, L.; Aquino, A.J.A.; Vazdar, M.; Jurkiewicz, P.; Pittner, J.; Hof, M.; Lischka, H. Absorption and fluorescence of PRODAN in phospholipid bilayers: A combined quantum mechanics and classical molecular dynamics study, J. Phys. Chem. A 2010, 115(41), 1142811437.

(11) Suga, K., Umakoshi, H. Detection of nanosized ordered domains in DOPC/DPPC and DOPC/Ch binary lipid mixture systems of large unilamellar vesicles using a TEMPO quenching method. Langmuir 2013, 29, 4830-4838.

(12) Hirose, M., Ishigami, T., Suga, K., Umakoshi, H. Liposome Membrane as a Platform for the 1-Pro-Catalyzed Michael Addition of trans- $\beta$-Nitrostyrene and Acetone. Langmuir 2015, 31(47), 12968-12974.

(13) Walde, P.; Ichikawa, S. Enzymes inside lipid vesicles: Preparation, reactivity and applications. Biomol. Eng. 2001, 18, 143-177. 
(14) Filippov, A., Orädd, G., Lindblom, G., Sphingomyelin structure influences the lateral diffusion and raft formation in lipid bilayers. Biophys. J. 2006, 90, 2086-2092.

(15) Barriga, H.M.G., Parsons, E.S., McCarthy, N.L.C., Ces. O.; Seddon, J.M.; Law, R.V., Brooks, N.J., Pressure-temperature phase behavior of mixtures of natural sphingomyelin and ceramide extracts. Langmuir 2015, 31, 3678-3686.

(16) Montich, G.G.; Cosa, J.J.; Maggio, B. Interaction of 1-anilinonaphthalene 8-sulfonic acid with interfaces containing cerebrosides, sulfatides and gangliosides, Chem. Phys. Lipids 1988, 49(1-2), 111-117.

(17) Gryczynski, I.; Malicka, J.; Shen, Y.; Gryczynski, Z.; Lakowicz, J.R. Multiphoton excitation of fluorescence near metallic particles: Enhanced and localized excitation, J. Phys. Chem. B 2002, 106(9), 2191-2195.

(18) Uchiyama, S.; Matsumura, Y.; De Silva, A.P.; Iwai, K., Fluorescent Molecular Thermometers Based on Polymers Showing Temperature-Induced Phase Transitions and Labeled with Polarity-Responsive Benzofurazans, Anal. Chem. 2003, 75(21), 5926-5935.

(19) Birks, J. B. Photophysics of Aromatic Molecules; Wiley-Interscience, New York, 1970.

(20) David, W.N. Biosynthesis of cholesterol and other sterols. Chem. Rev. 2011, 111, 64236451. 